Brit. J. vener. Dis. (1957), 33, 196.

$\checkmark$

\title{
SYMPOSIUM ON TRICHOMONAL INFESTATIONS, 1957
}

A meeting, described as the first European symposium on the subject of trichomonal infestations, held at Rheims from May 28-30, 1957, under the auspices of the French Society of Gynaecology, was attended by the delegates from approximately twenty countries, including some from North and South America. The subjects represented were gynaecology, urology, venereology, parasitology, epidemiology, hygiene, and veterinary medicine. Shortly before the Congress was due to begin, the participants received a printed volume containing more than forty articles in a diversity of languages, with summaries of variable quality in French, English, and German. An interesting feature was the inclusion of a reproduction of part of the original paper of 1936 , in which Donné described the discovery of Trichomonas vaginalis. It was assumed that those attending the congress had become acquainted with the contents of this volume and time was saved by taking these various papers as read. Meetings were devoted to discussion, the reading of papers which had not arrived in time for inclusion, and excellent final summaries by Dr. G. Chappaz, secretary and rapporteur général of the Congress. The purpose of the symposium, as declared by the President, Dr. A Netter, in his opening address, was to bring together leading investigators in the subject to enable them to discuss the problems involved and, as far as possible, to reach conclusions.

The proceedings began with a discussion on terminology. There seemed general agreement that the operative word should be Trichomonas and not Trichomonias, as some veterinary experts seemed to wish. The term "infestation" was preferred to "infection" because the organism is a protozoon. The accepted term, Trichomonas vaginalis, was criticized on the ground that infestation is not limited to the vagina, and the term Trichomonas Donné was recommended as a substitute. The meeting compromised on the description Trichomonas vaginalis Donné. There was some variation of opinion whether trichomonal infestation is becoming more common. The general conclusion was that trichomoniasis has always been very prevalent, but appears to be more common now because of better diagnostic methods and because other genital infections have become less widespread.
After a discussion on trichomoniasis in females, it seemed to be generally agreed that the evidence as to the frequency and importance of infestation of the urinary tract, a possible source of recurrences of vaginitis, was overwhelmingly strong. The importance was emphasized of symptoms of genital infestation other than vaginal discharge, namely pain, dysmenorrhoea, dyspareunia, urinary symptoms, and, particularly, various neuroses. Some speakers stressed the frequent association of mycotic infections with trichomoniasis, especially in pregnant patients. It seemed that trichomonal vaginitis of some duration produced changes in the epithelium covering the cervix uteri which could be mistaken for epithelioma. It was recommended that scrapings or biopsy specimens from this area should not be interpreted from the point of view of malignancy until treatment had cleared up the trichomonal inflammation.

With regard to trichomoniasis in the male, it was agreed that the condition was commoner than had been supposed in the past and that it was often difficult to diagnose. There seemed some division of opinion between French and British workers as to the method of diagnosis by smears. The French favoured stained smears and the British fresh smears obtained by scraping the urethral epithelium, but both were agreed as to the importance of cultures. There was little information as to the extent of the spread of the trichomonal parasite in the male genito-urinary tract, but Dr. Keutel (Berlin) showed some interesting sections of epididymes, obtained by epididymectomy, in which trichomonal forms could be identified within the tubules.

There was considerable discussion and some disagreement as to whether or not trichomoniasis can be regarded as a venereal disease. After a commission had been appointed to continue this discussion and formulate proposals, it was finally agreed that trichomoniasis is usually transmitted sexually. On this account it was recommended that public health authorities should regard it as equally important as other communicable diseases in the allocation of funds for control and for the purposes of research, but that they should not issue public announcements on this subject which might cause alarm and distress to those suffering from the condition. As far as possible, sexual partners should 
be treated to prevent re-infestation, but care should be taken not to cause distress and anxiety to patients by using the term "venereal" or emphasizing the fact of coital transmission. From the discussion on pathology it seemed that there was no evidence of a cystic or other resting form of this organism and there was no support for the theory of intestinal origin of the disease.

Discussions on treatment were hampered by differing assessments of cure. As Dr. Kupferberg (U.S.A.) pointed out, suppression of symptoms can be achieved by many so-called remedies, but to pronounce cure it is necessary to establish by the best possible methods, including careful, repeated search of microscopic specimens and first-class cultural technique, that the organism has been eliminated. The less critical the assessor the better the results of the treatment appear, and the better the diagnostic methods the less satisfactory the outcome of any treatment. The consensus of opinion was that, judged by the best standards, all forms of treatment were more often suppressive than curative. Aminitrazole was effective in the test tube but ineffective clinically in the recommended dosage. Regret was expressed that it had been so widely advocated without sufficient critical assessment. The greatest diversity of experience was evident in respect of the Japanese antibiotic Trichomycin. The Japanese delegation, headed by
Dr. Masanao Magara (Tokyo), claimed remarkable results with this drug, administered by mouth, in the treatment both of trichomoniasis and of Candida infections, but other workers present described very disappointing results. In his final summary, Dr. Chappaz suggested that this difference of experience might be due to the fact that European workers had used enteric-coated capsules, whereas the Japanese used the powdered drug suspended in water. It was possible that the enteric coating delayed or prevented absorption.

On the social side the Congress was an immense success owing to the kindness and imaginative hospitality of our French hosts. A conducted visit to the famous Cathedral at Rheims, a reception by the Mayor of the City at the Hôtel de Ville where the meetings were held, a banquet by candle-light in the champagne cellars of Ruinart, an evening with scientific films and, later, champagne at the Hôtel des Comtes de Champagne, and the final generous gesture of the French Society of Gynaecology, a tour through the vineyards of the renowned Route de Champagne followed by lunch at Maison Moet et Chandon and visits to the vineyards Clos des Giosses and the Caves Mercier-all were occasions which went with a swing and involved the consumption of liberal and delightful quantities of the local vintages.

A.J.K. 